\title{
Pragmatisme et responsabilité sociale de l'entreprise
}

D'une manière similaire aux recherches considérant la nature problématique de l'hypothèse de l'individualisme atomisé, ce travail estime que la RSE est le fruit de l'interaction d'acteurs sociaux et non atomisés. Cet article souligne les insuffisances des approches théoriques majeures en proposant une autre voie comme posture philosophique et théorique à l'analyse de la RSE. Il étudie ensuite le mode opératoire développé par les acteurs, dans leur interaction et face aux problèmes rencontrés, pour construire la RSE. Quatre études de cas mettent en évidence la façon dont les acteurs traitent la résolution d'une problématique en RSE par des processus d'apprentissage et de créativité. Les valeurs opérationnelles et terminales de la RSE émergent au fur et à mesure des apprentissages réalisés, en interaction avec l'ensemble des parties prenantes concernées par le problème à résoudre, sous forme de solutions innovantes. 
$\mathrm{L}$ a responsabilité sociale de l'entreprise (RSE) - entendue comme englobant les attentes économiques, légales, éthiques et discrétionnaires (ou philanthropiques) de la société à l'égard des organisations à un moment donné (Carroll, 1979, 2004) - fait l'objet depuis un certain nombre d'années d'âpres débats entre chercheurs, praticiens, État, organisations non gouvernementales et autres acteurs de la société contemporaine. Tous ces acteurs s'interrogent sur le rôle, voulu élargi, de la firme dans la société et sur les réponses qu'elle doit fournir à la création de richesses et à la satisfaction des multiples attentes. Ils veulent ainsi récuser l'approche contractuelle de la firme (Donaldson, 1999; Jensen, 2002), qui considère que l'unique objectif de l'entreprise consiste à atteindre la maximisation de la richesse au bénéfice de l'actionnaire et questionne la valeur et la légitimité de tout traitement des problèmes sociaux ou sociétaux par la firme. Ces débats ont conduit à la prolifération de travaux substantiels ayant permis des développements théoriques et conceptuels et l'exploration empirique des comportements des organisations, l'objectif étant de démontrer que la prise en compte de la RSE n'est guère incompatible avec la maximisation de la richesse. Cependant, ces contributions, en dépit de leurs apports indéniables à l'avancée de la réflexion dans le champ de la RSE, sont restées tributaires de l'hypothèse de l'individualisme atomisé (Buchholz et Rosenthal, 2001), issue de la pensée moderne. Cette hypothèse laisse entendre que l'individu est le socle de la société, et que celle-ci n'est rien d'autre que la somme des individus. Dans cette perspective, les organisations sont considérées comme des entités indivi- dualisées et séparées de la société, opérant dans un environnement concurrentiel, afin de produire des biens et des services, et cherchant à optimiser la création de leurs richesses. À cause cependant de l'impact social, supposé ou réel, de leurs activités, ces entités ne peuvent pas se soustraire de leurs supposés devoirs à l'égard de la cité.

L'adoption explicite ou implicite de cette hypothèse par les différentes contributions rend mitigées les réponses apportées aux problématiques sociales et sociétales dans la mesure où les unités en considération, isolées, indépendantes, demeurent dans une tension irréconciliable et ne peuvent pas fonder, par conséquent, une véritable société ou communauté. Cette conception, conduisant à considérer les systèmes de valeurs d'une manière exogène - les comportements des organisations sont appréciés à l'aune d'un système de valeurs transcendantal -, en fonction du rapport de forces du moment, reste ancrée, d'une manière surprenante, dans la vision contractuelle de la firme. Comment faire alors lorsqu'il importe de considérer tout à la fois l'efficience économique, la maximisation de la richesse, l'amélioration sociale et environnementale, les aspects humains, les droits des actionnaires ainsi que ceux de toutes les autres parties prenantes concernées par l'activité des firmes? Et comment prendre en compte et gérer les arbitrages, les conflits et les dilemmes, sous-jacents aux attentes antinomiques?

À l'instar des recherches considérant la nature problématique de l'hypothèse de l'individualisme atomisé (Buchholz et Rosenthal, 2001; Solomon, 1992; Wicks et al., 1994), ce travail questionne le rôle de la firme dans la société en estimant que la responsabilité sociale de l'entreprise (RSE) 
est plutôt le fruit de l'interaction d'acteurs sociaux. N'empruntant pas la voie de la synthèse entre la RSE et l'approche économique et contractuelle de la firme, cette recherche part de la tension entre l'économique et le social, traite de la collision des différents objectifs et intérêts (Alexander, 1988 ) et suggère le pragmatisme (James, 1975) comme approche permettant d'appréhender le rôle approprié et élargi des firmes dans la société et de saisir les différentes réponses qu'elles apportent aux questions sociétales.

Dans cette perspective, la RSE est considérée évolutive en fonction de l'expérience vécue. Elle n'est pas uniquement le résultat de l'optimisation de critères de choix. La RSE n'est pas non plus une réalité à atteindre (un idéal type) mais une construction et le résultat d'expérimentations. La mise en œuvre de la RSE peut être appréhendée comme un parcours initiatique de l'organisation (des acteurs) avec ses parties prenantes pour construire une identité sociale légitime.

Aussi nous proposons-nous, dans les propos qui suivent, de réorienter la perspective de la RSE, par l'adoption de la posture du pragmatisme, via le questionnement sur le rôle de l'entreprise dans la société. Comme la démarche pragmatiste nécessite la description systématique des expériences vécues, le second objectif est de montrer que les récits de vie constituent une méthode appropriée pour apprécier les réponses fournies par les organisations aux questions sociétales. À cet égard, le mode opératoire développé par les acteurs, dans leur interaction et face aux problèmes rencontrés, pour construire des pratiques perçues comme socialement responsables, est étudié au travers de quatre études de cas appréhendées au moyen de la méthode des récits de vie.

Trois parties structurent cet article. La première partie traite du pragmatisme, comme voie théorique pour étudier la RSE. La deuxième partie porte sur le champ d'observation et sur la méthodologie choisie. Il s'agit en l'occurrence de quatre études de cas appréhendées au moyen de la méthode des récits de vie. Enfin, la troisième partie présente et discute les résultats obtenus par la recherche ainsi que des implications qu'ils entraînent.

\section{LA VOIE DU PRAGMATISME POUR ÉTUDIER LA RSE}

La thèse de cette recherche est de considérer que la philosophie pragmatiste constitue un système approprié pour l'étude de la RSE et une voie fructueuse pour prendre en compte les organisations dans leurs relations avec la société (Buchholz et Rosenthal, 1997; Margolis, 1998). Le pragmatisme récuse ainsi les modèles économiques traditionnels, fondés sur l'individualisme atomisé ${ }^{1}$. Buchholz et Rosenthal (1997, p. 199) écrivent à cet égard: « la philosophie pragmatiste, fondée sur différentes hypothèses, ouvre la possibilité de développer une théorie alternative à la firme dans la société qui fusionne le marché et la politique publique, qui dépasse les dichotomies traditionnelles entre firmes et État, entre

1. Bien entendu, la critique de l'hypothèse de l'individu atomisé n'est pas le seul apanage du pragmatisme. Nous pensons notamment aux démarches cognitives à l'interactionnime symbolique illustré par les travaux de Weick (1995). 
l'économique et le social, entre le public et le privé ». Pour les besoins de cette recherche, trois axes du pragmatisme méritent d'être soulignés: le statut de la science et de la vérité dans le pragmatisme; l'éthique et la réunion du descriptif et $\mathrm{du}$ normatif; le rejet de l'hypothèse d'atomicité.

Le premier axe consiste à récuser la modernité du monde, contenue dans la philosophie cartésienne de la nature et de la science où l'objet scientifique est relatif à l'étude de la réalité sous forme d'unités séparées, isolées et objectivées, dont les principes de coordination sont mis à jour, au moyen de lois scientifiques, libres de tout système de valeurs. Est fait alors appel aux présupposés d'une théorie dite « spectatrice » de la connaissance, conduisant à une interprétation philosophique quelque peu naïve de la connaissance scientifique, qui fournit une description littérale du fait objectif en excluant de facto l'expérience qualitative, comme chemin d'accès à la réalité. Prédomine dans cette acception la définition quantitative de l'univers, avec, comme corollaire, la séparation de l'esprit et de la matière, l'aliénation de la nature et des humains, ainsi que la séparation des humains des communautés dans lesquelles ils vivent et développent leur " moi » (Dewey, 1958).

Face à cela, la philosophie pragmatiste considère que des expériences testées et éprouvées par les hommes conduisent à la connaissance en inférant des relations causales entre des événements externes et partant, en développant l'interprétation et la compréhension de l'environnement (Wicks et Freeman, 1998). Chaque individu est ainsi porteur de science et bien que les chercheurs académiques professionnels pratiquent l'expérimentation rigoureuse et formalisée, les individus ordinaires apprennent également de leurs expériences.

À partir de là, la compréhension des fondements du monde passe par le rejet de toute notion de réalité transcendantale, coupée de l'expérience vécue des individus, et dont l'accessibilité n'est possible qu'au moyen de la raison pure (Dewey, 1958; Joas, 1993, 1996). L'existence d'une réalité partagée met les individus sur un plan commun dans lequel la communication et la coopération (et donc les pratiques des entreprises) peuvent être considérées (Dewey, 1958, p. 74). Le refus d'une réalité transcendantale et réifiée conduit les pragmatistes à estimer le caractère non absolu de la vérité, celle-ci ne pouvant être que provisoire et instrumentale et liée à l'expérience. À cet égard, il peut être estimé qu'en matière de RSE, les organisations peuvent jouer un rôle efficace dans le traitement de l'ensemble des questions sociétales et ne doivent pas se cantonner à un rôle d'optimisation de la création de richesses. De cette idée, le pragmatisme conduit à s'interroger sur les conséquences de l'action sur la pensée. Les firmes fontelle réellement une différence concrète dans le traitement des différents problèmes de type social? La réflexion devrait alors porter sur la mise en évidence des effets induits par les actions pour traiter des problèmes sociétaux. Par ailleurs, admettre que les firmes doivent jouer un rôle tant social qu'économique mène à s'interroger sur la façon dont cette idée doit être appliquée et sur l'obtention concrète de différences entre les dimensions économiques et les dimensions sociales. Ceci donne une autre perspective à la RSE. Quelles sont dans cette optique les conditions et les processus per- 
mettant aux entités visées de bénéficier des actions des organisations?

Le deuxième axe consiste à confondre la description et la prescription. En effet, évoquant l'éthique et son caractère désincarné, Dewey (1920, p. 173) écrit: " Quand tout est dit et fait pour critiquer les déficiences sociales actuelles, on peut bien se demander dans quelle mesure la difficulté fondamentale ne se trouve pas dans la séparation de la science morale de la science naturelle. » Partant, le pragmatisme considère que l'expérience est le fondement de l'éthique et propose une science éthique basée sur le test d'hypothèses. Ainsi, les individus construisent des jugements hypothétiques sur leurs comportements prospectifs, anticipent l'interaction des comportements proposés avec l'environnement social et physique et en tirent des conséquences en matière de détermination de leurs croyances. Le pragmatisme conçoit ainsi la notion d'obligation (et partant de la responsabilité) comme déterminée socialement et par l'expérience. Il récuse dans cette perspective la logique sous-jacente de l'impératif catégorique de Kant, en l'occurrence que les nouvelles situations morales doivent être jugées sur la base d'un ensemble de règles prédéfinies. Il souligne par ailleurs le mérite de l'approche utilitariste focalisée sur l'analyse des conséquences des actes, tout en remettant en cause la manière méca- nique et quantitative avec laquelle Bentham (1834) appréciait les conséquences (par le biais du comptage d'unités de bonheur).

L'approche pragmatiste nécessite une analyse précautionneuse et au cas par cas. Chaque cas est ainsi source d'apprentissage et tout enseignement issu du cas permet des économies de recherche et d'efforts pour les situations subséquentes. Dans cette optique, l'individu considère une situation donnée et la place dans un contexte instructif, suggérant une réponse possible. Parfois, on choisit une analogie appropriée et on apprend des conséquences de ce choix: ce qui demeure fondamental, c'est le jugement $^{2}$.

Dès lors, il est possible de construire une approche pragmatiste du choix moral, qui requiert que les individus considèrent les conséquences sociales (immédiates et émergentes) de leurs actions. Développement personnel, apprentissage issu de l'expérience et personnalité permettent l'occurrence de cette évaluation.

Sur le plan de la RSE, nous admettons dans cette recherche que la firme est un acteur social, sans octroyer à cette hypothèse un caractère normatif figé, et sans rejeter les autres croyances sous-tendant l'existence des organisations. En effet, tant l'approche de type friedmanien (1962) selon laquelle la société est mieux satisfaite si les firmes visent d'une manière exclusive la maximi-

\footnotetext{
2. Cette présentation du choix moral peut ressembler de prime abord à la décision rationnelle, telle qu'elle est développée par Simon (1979). En réalité, il n'en est rien car l'approche des pragmatistes est différente en ce sens qu'elle aborde une situation morale, non un problème technique, qu'elle légitime des émotions et qu'elle est itérative. Le pragmatiste peut rappeler également la théorie de la contingence (particulièrement l'approche de Follett, voir à ce sujet Wren et Greenwood, 1998). Cependant, les théories de la contingence recommandent des approches de management spécifiques qui sont en adéquation avec la réalité d'une organisation donnée (ce qu'appelle la loi de la situation). La théorie de la contingence, de nature managériale, peut ainsi justifier un style de management donné. D'un autre côté, le pragmatisme suppose une conscience étendue des conséquences sociales et vise à trouver des solutions pour des problèmes affectant la société dans son ensemble et pas seulement des solutions pour des problèmes immédiats ou ceux de l'organisation.
} 
sation des richesses que celle qui intègre l'ensemble des parties prenantes (Freeman, 1984; voir également Gond et Mercier, 2005, pour une synthèse sur la littérature relative aux parties prenantes) sont retenues. La démarche scientifique consiste fondamentalement à mettre à l'épreuve une pensée pragmatique pour déterminer si l'action menée à partir de la croyance choisie produit les conséquences désirées. La manière dont ces conséquences doivent être pondérées ou abandonnées par rapport à d'autres relève du choix normatif. Dès lors, il importe d'étudier la RSE en explorant ce qui se passe quand les couples efficience instrumentale/bien-être humain, maximisation de la richesse/amélioration des conditions sociétales, droits des actionnaires/droits des parties prenantes sont tous pris en compte. $\mathrm{La}$ démarche suggérée reconnaît ces conceptions antinomiques et intègrent les tensions éventuelles. Ce qui signifie qu'il faut s'appuyer sur une approche descriptive pour explorer les réponses des firmes aux questions posées et sur une analyse philosophique rigoureuse, conduisant à l'émergence d'une approche normative de l'entreprise.

Le troisième axe porte sur le refus de la dichotomie traditionnelle entre l'individu et la société. Ainsi, l'expression ou la communication des idées ne peut se réaliser qu'en faisant référence aux groupes dans lesquels l'identité des individus a évolué et émergé. Les relations entre les organisations sont analogues, dans la conception des pragmatistes, à celles liant le moi et la communauté. Aucune organisation ne peut dès lors exister isolément de la société ou de son environnement, et la société est en relation avec les institutions la constituant. L'entre- prise est par conséquent un objet social, agissant dans la société, au contact d'autres agents opérant de manière identique, et non une entité atomisée (Stern et Barley, 1996). La conséquence de cette conception est que les organisations constituent une part importante de la société et fournissent les sens et les valeurs qui l'alimentent. Mais ce sont des parts inséparables de la société, des unités non isolées ayant certains types d'obligations envers la société. Cette vue conduit à penser que la responsabilité de l'entreprise dans la société serait plus appropriée pour le champ que la notion de RSE.

De ce qui précède, nous considérons que les organisations et les individus, acteurs sociaux, construisent la RSE dans leur interaction, au moyen de leurs expériences et de leur vécu. Certes, l'idée de la RSE peut exister comme idée ou concept ex ante, mais ce n'est qu'une hypothèse ou un plan d'action. Sa mise en œuvre comme concept est en même temps sa mise à l'épreuve. Il importe dès lors d'étudier, dans une perspective descriptive, comment les acteurs fondent leurs comportements, comment ils envisagent les différentes réponses, comment ils évaluent et sélectionnent ces réponses, comment ils les mettent en œuvre, et enfin, quelles sont les conséquences de ces tentatives en termes de RSE. Par ailleurs, comme la perspective descriptive adoptée vise à mettre à jour les actions entreprises, il importe, dans une démarche normative, d'identifier les raisons sous-jacentes aux choix effectués et de clarifier les considérations antinomiques.

Dans ce qui suit, nous présenterons et justifierons le champ d'observation sur lequel s'appuie cette recherche et nous exposerons la méthodologie adoptée. 


\section{CHAMP D'OBSERVATION ET MÉTHODOLOGIE}

L'objectif de cet article étant d'établir comment et pourquoi les acteurs construisent au fur et à mesure des expériences vécues et en interaction avec leur environnement la RSE, la parole doit être donnée aux acteurs. Il s'agit notamment d'identifier des individus ayant joué un rôle moteur dans la résolution de problématiques en RSE et ayant participé activement à la mise en œuvre d'actions en RSE. La recherche s'appuie sur une étude de la narration des acteurs pour faire émerger les processus par lesquels les individus envisagent la résolution de problèmes de RSE dans un contexte donné.

\section{Terrain d'observation}

La recherche du terrain consistait à identifier des informateurs ayant acquis une expérience en matière de réflexion et de pratiques de la RSE. La condition première assurant la qualité et la richesse des données étant la relation de confiance qui s'établit entre le sujet et le chercheur, le choix a porté sur des individus avec lesquels des liens étaient établis du fait de rencontres précédentes ou de connaissances communes. Quatre terrains d'investigation (décrits succinctement ci-dessous et exposés dans le tableau 1) ont ainsi été retenus.

\section{$1^{\text {er }}$ cas. Marque verte}

En 1996, BM est recruté pour créer un poste d'ingénieur environnement sécurité au sein d'une fédération professionnelle pour encourager la prise de conscience des entreprises de la branche sur ces thématiques alors peu prises en compte. En 1999, un incident de pollution met en cause les entreprises membres de la fédération et provoque une pression importante de la part de l'agence de l'eau et la ville sur la chambre des métiers pour encourager un changement de pratiques. Cet incident, inscrit dans un environnement global - budget ministériel, réglementation, concurrence internationale, pression des collectivités et des fournisseurs, problème d'image des imprimeurs - favorable au développement des pratiques environnementales, justifie l'engagement de BM dans la démarche de création d'une marque professionnelle « écologique ». La démarche n'a pas été acceptée aisément au départ, mais au fur et à mesure des opportunités et des expériences, la méthode est affinée et $\mathrm{BM}$ réussit à faire adopter «la marque verte » par les membres de la fédération. Cependant, le développement connaît des limites et aujourd'hui de nouvelles solutions sont recherchées pour atténuer ses insuffisances, voire les éliminer. En outre, suite à différentes rencontres avec des acteurs extérieurs (collectivités, certificateurs, fonctionnaires ministériels) et à l'analyse de l'évolution de la réglementation en termes de prévention des risques, $\mathrm{BM}$ entrevoit de nouvelles manières d'ancrer l'entreprise de son secteur dans la société notamment avec la volonté d'assurer un contrôle plus strict des pratiques environnementales et d'associer à celles-ci de bonnes pratiques sociales. Aujourd'hui, un projet de label est ainsi en cours de validation, dans l'attente d'un accord politique.

\section{$2^{e}$ cas. Plate-forme de déchets}

Suite à une rencontre avec un collègue ayant mis en place de la veille environnementale, FA identifie des sources de coûts 
futurs risquant de peser lourdement sur les entreprises, notamment les très petites entreprises (TPE) n'ayant pas anticipé l'évolution de l'environnement réglementaire et les pressions institutionnelles. FA cherche à concilier performance économique et lien avec la cité. Cette démarche vise à résoudre un dilemme entre la vision négative de l'entreprise portée par ses parents et son désir d'être entrepreneur. Elle est ainsi guidée par la croyance selon laquelle l'entreprise n'est performante économiquement que lorsqu'elle s'inscrit dans la cité, en établissant des liens sociaux et de proximité. En étudiant les textes réglementaires, en rencontrant différents acteurs agence de l'eau, collectivités, autres entreprises, fédérations professionnelles - FA s'est progressivement rendu compte que le problème principal de mise en œuvre de bonnes pratiques environnementales est dû au fait que les acteurs agissent dans des mondes séparés tout en étant contraints les uns par les autres. En outre, elle entrevoit dans la mise en œuvre d'une solution à ces problèmes, un gisement d'emplois et une activité rentable pour tous les acteurs. C'est ainsi que petit à petit est monté le projet de plate-forme de déchets.

\section{$3^{e}$ cas. Maison des consommations citoyennes}

Jeune diplômé, MT sensible aux problématiques de discriminations raciales tente d'y remédier au sein de son école de commerce puis au sein de son entreprise de conseil dès son recrutement. Cette démarche lui vaut dans le cadre professionnel des problèmes relationnels allant jusqu'au risque de licenciement. Depuis, travaillant dans une ambiance plus en phase avec ses convictions en termes de valeurs, MT cherche à mettre en œuvre ses préoccupations en termes de RSE en dehors de l'entreprise dans laquelle il estime ne pas avoir de marge de manœuvre. Il est ainsi aujourd'hui administrateur au sein de deux organisations: une association de soutien (en conseil et communication) aux ONG et le montage d'une maison des consommations citoyennes. Le second projet vise la réalisation d'un lieu référent donnant l'exemple d'une activité économique rentable respectant les critères sociaux et environnementaux.

\section{$4^{e}$ cas. Agence de communication}

Technicien dans le domaine publicitaire, EG vouait dès son jeune âge une admiration pour la réalisation télévisuelle. Son parcours a débuté auprès d'un « génie » de la publicité qui lui a donné le plaisir de la création artistique. Cependant, au fur et à mesure de son expérience professionnelle et de rencontres, il s'interroge sur la finalité de son métier en termes de sens pour la société. Deux événements - maladie, licenciement - marquent une rupture dans son cheminement et le poussent à remettre en question le système dans lequel il a évolué. À la recherche d'une solution lui permettant de concilier ses valeurs et ses compétences, il crée une agence de publicité visant à « fabriquer de la communication » en redonnant à la publicité sa fonction d'outil au service de l'artisan agissant en société.

\section{Mode de collecte de l'information: les récits de vie}

Considérant les limites des recherches antérieures en termes de compréhension de la RSE, la présente réflexion se concentre sur l'étude du sens donné par les acteurs à la mise en œuvre de solutions en RSE. Dans 
Tableau 1 - Présentation des cas étudiés

\begin{tabular}{|c|c|c|}
\hline Personnes interrogées & Entreprise d'origine & Projet en RSE \\
\hline $\begin{array}{c}\text { (1) Ingénieur en } \\
\text { développement durable }\end{array}$ & $\begin{array}{c}\text { Fédération professionnelle } \\
\text { des imprimeurs }\end{array}$ & Marque verte \\
\hline (2) Chef d'entreprise & Entreprise de services & $\begin{array}{c}\text { Association d'insertion } \\
\text { professionnelle/ } \\
\text { Plate-forme déchets }\end{array}$ \\
\hline (3) Consultant & Entreprise de conseil & $\begin{array}{c}\text { Maison de la consommation } \\
\text { citoyenne }\end{array}$ \\
\hline (4) Publicitaire & Agence de publicité & Création d'une agence alternative \\
\hline
\end{tabular}

une approche pragmatiste mettant l'accent sur l'interprétation donnée par les acteurs à leurs pratiques, la collecte de la parole de ces derniers semble nécessaire. Dans ce contexte, la méthode des récits de vie paraît particulièrement appropriée. Il s'agit d'une méthode de recherche interdisciplinaire en sciences sociales. Récente en sciences de gestion, la méthode a été développée dans les années 1980, pour analyser notamment les pratiques des organisations (Christian et Boudès, 1998; Giroux, 2000). Définis comme l'expression générique où une personne raconte sa vie ou un fragment de sa vie à un ou plusieurs interlocuteurs (Legrand, 1989), les récits de vie résultent d'une forme particulière d'entretien - l'entretien narratif - au cours duquel le chercheur demande au sujet désigné de lui raconter son expérience vécue (ou une partie de cette expérience).

Plus précisément, le récit de vie est un discours narratif dans lequel une histoire « réelle » est improvisée au cours d'une relation dialogique. Il ne vise pas à comprendre un individu donné mais un frag- ment de réalité sociale et historique, un objet social.

Dans le cadre des récits de vie, les individus sont considérés comme des acteurs sociaux non passifs. Comme les comportements ne résultent pas de jeux déterministes ou de stimulus réponse, les récits sont un moyen d'accéder aux processus internes des sujets, tant sur un plan psychologique que social. Cette méthode permet d'appréhender le système évolutif de normes qui régulent l'action et qui en résultent. Il s'agit alors de dresser un schéma d'ensemble qui prend en compte les projets des sujets étudiés et le sens donné à leur responsabilité de même que le rôle qu'ils s'attribuent. Analyser la RSE par le récit que les acteurs en font est une démarche de recherche qui permet de décrire, comprendre et analyser « l'agir en situation »(Bertaux, 1997). Et de saisir, comme le stipule la philosophie pragmatiste, les articulations entre les phénomènes objectifs, les déterminations inconscientes et les expériences subjectives. 
Aussi l'entretien de nature semi-directive vise-t-il à faire raconter les expériences vécues par les acteurs interrogés avec des questions du type "Racontez-nous comment vous avez monté votre projet en RSE. » « Comment faites-vous pour la mettre en œuvre?».

En outre, faisant appel à l'intrigue - un projet à réaliser, une action à accomplir, un problème à résoudre - qui met en scène des personnages, des décors et des situations, la forme narrative possède un pouvoir d'évocation qui permet de saisir comment les individus construisent pour eux-mêmes et pour les autres la signification de leur monde. Cette méthode est dès lors particulièrement bien adaptée pour traduire la temporalité de la notion de RSE voulue ou mise en œuvre par les acteurs. Cette temporalité est saisie au travers de questions du type « racontezmoi l'histoire de votre projet en RSE, comment et pourquoi votre projet a-t-il évolué? ».

Ensuite, la mise en récit joue le rôle de mémorisation et d'institutionnalisation de l'expérience commune. Ce faisant, elle participe des mécanismes d'interprétation collective qui sont au cœur des fonctionnements des organisations (Daft et Weick, 1994) et offre la possibilité de comprendre la construction réalisée dans l'interaction: «Avec qui avez-vous mis en place ces solutions? ». Dans ce cadre, le guide d'entretien est contingent au sujet étudié. Les récits de vie se révèlent ainsi comme une méthode permettant d'envisager l'étude de la responsabilité dans un cadre plus large intégrant l'arrière plan culturel, les événements personnels, les oppositions et les argumentations de l'acteur.

\section{Méthode d'analyse des récits : posture analytique}

Trois postures (Demazière et Dubar, 1997) peuvent être adoptées pour mener l'analyse d'un récit de vie. La posture illustrative, dans une logique causale, vise à mettre en évidence des relations entre un objet et des variables indépendantes. C'est plus le contenu que la structure du discours qui est étudié. La posture restitutive porte sur une démarche hyper-empirique selon laquelle l'individu est acteur du social et producteur de ses comportements. Il s'agit ici de décrire cette réalité dans son contexte spatiotemporel. Enfin, la posture analytique cherche à reconstruire le sens à partir de l'analyse des entretiens. Compte tenu de leurs objets respectifs, les deux premières postures ne semblent pas convenir à l'objet de la présente recherche. En effet, la posture illustrative fondée sur l'analyse de contenu présente la faiblesse de « détacher les passages de leurs contextes discursifs » et d' « appauvrir voire de modifier le sens » (Bertaux, 1997, p. 91-92). La posture restitutive, parce qu'elle fait abstraction de la relation entre le narrateur et le chercheur, ne permet pas de faire apparaître le processus de construction du sens.

Dans le cadre de cette recherche, comme l'objet de l'étude est de comprendre les mécanismes et processus déployés par les individus pour construire la RSE, la posture analytique paraît être la plus pertinente. Centrée sur le sujet avec lequel s'est établie une relation de confiance, cette posture suppose un travail très méthodique d'interprétation du discours pour produire et/ou développer la théorie. Elle a pour principal objet de mettre à jour le processus d'appropriation des formes sociales. 
Les développements qui suivent sont consacrés à l'analyse et l'interprétation des données collectées en vue de répondre à l'objectif de cette recherche, consistant à montrer comment la RSE est construite par les acteurs sociaux au cours de leurs interactions et sur la base de leur vécu et de leurs expériences.

\section{ANALYSE ET DISCUSSION DES RÉSULTATS ET IMPLICATIONS DE LA RECHERCHE}

Afin de construire les schèmes des entretiens, la structure du récit est analysée pour mettre en évidence la logique de construction sociale sous-jacente. Il s'agit d'identifier pour chacun des entretiens les liens de disjonctions et de conjonctions entre catégories de séquences, d'actants et d'arguments.

\section{Analyse de l'entretien de BM, marque verte d'une fédération professionnelle}

La partition la plus pertinente qui émerge ici est celle opposant les actions en RSE « volontaires » aux actions « imposées ». Les actions imposées par les collectivités ou la loi sont vécues comme une « perte de contrôle », mais peuvent devenir des « opportunités » pour les entreprises. $\mathrm{Si}$ l'on reste dans la situation de perte de contrôle, il peut y avoir alors mise en œuvre d'une RSE non voulue comme par exemple la certification ISO. Ce comportement opportuniste peut engendrer d'une part, des " comportements déviants" risquant de remettre en question le système RSE mis en place et aboutir à une perte de contrôle. D'autre part, une valorisation de l'action en RSE peut être obtenue en termes de marché, de climat social ou d'avantage concur- rentiel. Dans le cadre de la RSE issue d'une démarche volontaire, nous observons à la fois un comportement opportuniste (profiter de ressources, de lobbying, etc.), mais aussi une volonté de " prévenir les risques ", d'être dans une posture proactive pour contrôler les modes d'action. Dans cette optique, deux visions coexistent. La première s'inscrit dans la « culture actuelle » des acteurs, la RSE étant un moyen d'anticiper des actions coercitives ou de se prémunir contre des accidents engendrant alors une valeur à court terme pour les acteurs. La seconde prône un " changement de culture » allant jusqu'à la rupture pour promouvoir une action en RSE ayant une valeur durable pour les acteurs. La solution construite au fur et à mesure est alors de proposer la mise en œuvre d'un label de développement durable dans les services dont le maître d'œuvre serait la fédération professionnelle.

La conception de l'entreprise est l'élément structurant des propos de FA. L'entreprise peut ainsi « être hors de la cité » et alors ne pas jouer son rôle en termes de RSE car l'horizon de la responsabilité est limité par les actionnaires et se traduit par la recherche de performance financière essentiellement. Mais les valeurs du dirigeant peuvent l'amener à vouloir faire du bien et le décalage entre les impératifs économiques financiers et ses valeurs personnelles engendrent une situation de « schizophrénie ». L'entreprise « en société »a quant à elle comme objectif de rechercher la performance économique et sociale en respectant un nombre de règles minimum (intégrant les valeurs du dirigeant et des valeurs personnelles). Le problème de la mise en œuvre opérationnelle peut être facteur de passivité des entreprises. Ces der- 
Figure 1 - Schème de l'entretien 1 : la RSE comme vecteur de changement culturel de la profession

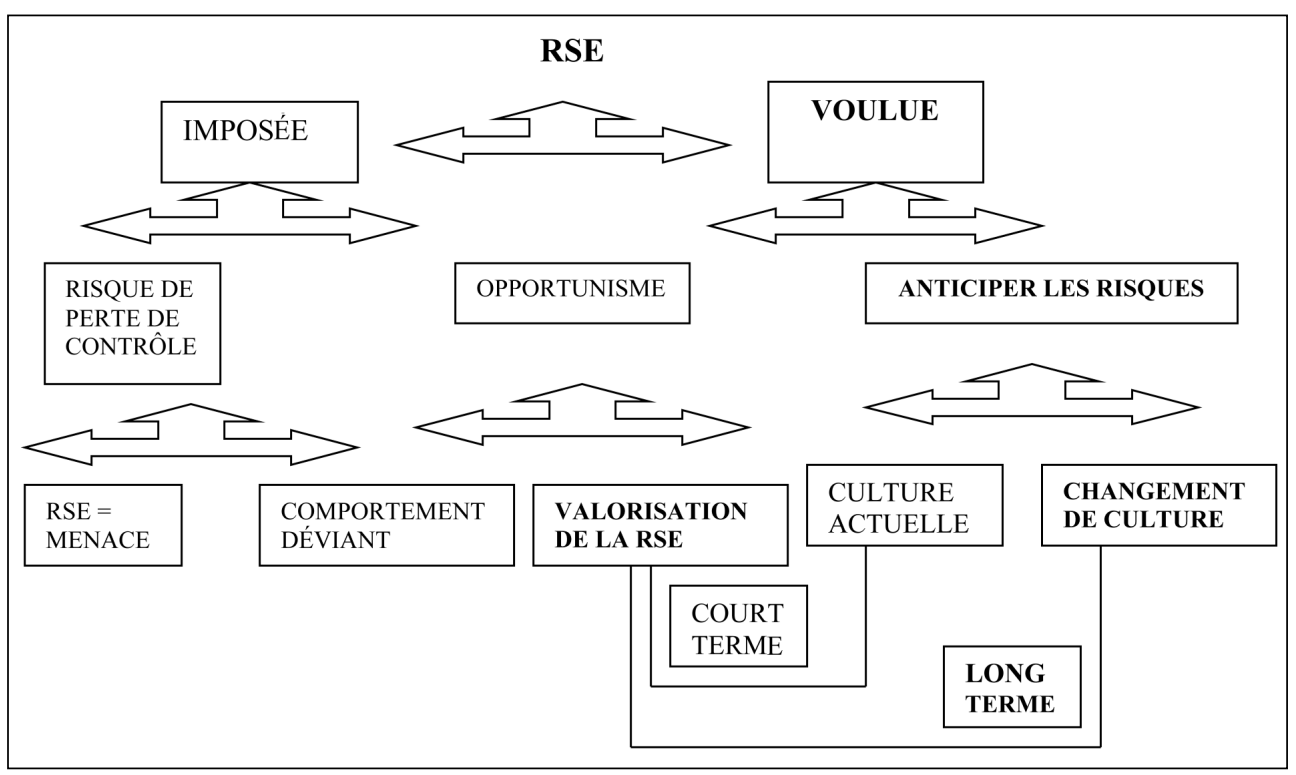

nières, en ne faisant pas le minimum requis pour être dans la cité, se mettent hors la loi. La mise en œuvre d'un savoir-faire en matière de RSE n'est cependant pas accessible à tous les acteurs, notamment de nombreux gaspillages de ressources (financières et de temps) sont réalisés sans résultat. Le savoir-faire en RSE ne peut être obtenu selon l'interviewé, qu'en interaction avec l'ensemble des parties prenantes concernées par la RSE et en créant une structure simplificatrice de la mise en œuvre et créatrice elle-même de valeur pour la société en termes économique et social. La responsabilité environnementale est alors un moyen de résoudre le dilemme de l'entreprise, acteur de la cité et citoyenne ou acteur hors cité.

La RSE est posée au travers de deux catégories définissant l'entreprise: d'une part, une vision dissociative, d'autre part, une vision intégrative. Dans le premier cas, l'entreprise n'est pas « mère Teresa », elle n'a pas à se préoccuper de morale, son rôle est d'être performante sur le plan économique. Mais, en même temps, cette démarche pose problème, compte tenu de l'impact négatif de l'entreprise aujourd'hui tant en termes social qu'environnemental. La solution peut être le mécénat mais si l'entreprise s'en contente, cette solution est réductrice. Globalement, l'entreprise est confrontée à un dilemme.

Dans une vision intégrative de l'organisation et des acteurs dans la société, le système de valeurs guide les comportements. L'absence de conscience morale entraîne des comportements non responsables. Face à la motivation de bien faire, l'acteur rencontre des contextes favorables à l'action en dehors de l'entreprise, sa position en tant 
Figure 2 - Schème de l'entretien 2 : faire de la RSE pragmatiquement pour que l'entreprise reste dans la cité

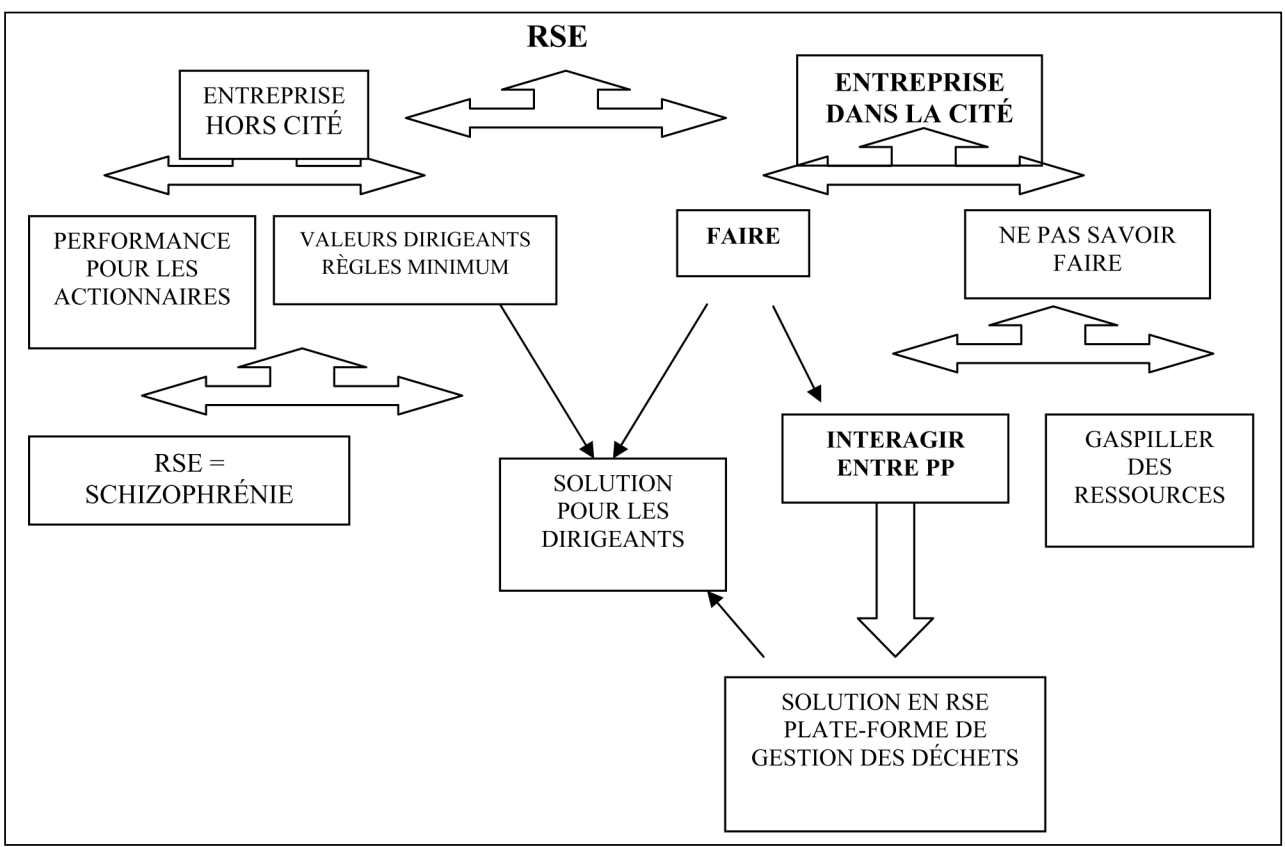

que salarié lui est en revanche défavorable pour pouvoir mettre en œuvre ce qu'il estime relever de la responsabilité de l'entreprise.

Face à ces obstacles internes, deux démarches sont possibles en dehors de l'entreprise: soit l'affrontement avec l'entreprise, soit la démonstration que la RSE rapporte - « le nerf de la guerre pour les entreprises, c'est le pognon $»-$. La solution est de réaliser la RSE en dehors de l'entreprise en intervenant dans des ONG (appuyer la communication, l'image des pratiques responsables) et en participant à la création d'une organisation exemplaire en termes de RSE et rentable de sorte à encourager des pratiques responsables des entreprises.
La définition du métier d'EG guide ses propos sur la RSE. Les agences peuvent faire de la publicité ou fabriquer de la communication. Quand elles font de la publicité, elles sont l'expression d'un génie créatif ou le reflet de la manipulation. Dans le premier cas, c'est le métier de l'artiste qui n'a pas grand chose à voir avec la RSE. Dans le second cas, cela dénote d'une vision courttermiste de l'entreprise dans la société qui ne cherche pas l'essentiel mais uniquement la performance économique pour faire acheter, créant ainsi du déséquilibre dans la société. En revanche, fabriquer de la communication revient à réaliser un travail d'artisan dont l'outil est la publicité. Celle-ci peut alors être créatrice de sens si elle se met au service d'organismes qui valorisent 
Figure 3 - Schème de l'entretien 3 : Valoriser par le marché la démarche en RSE

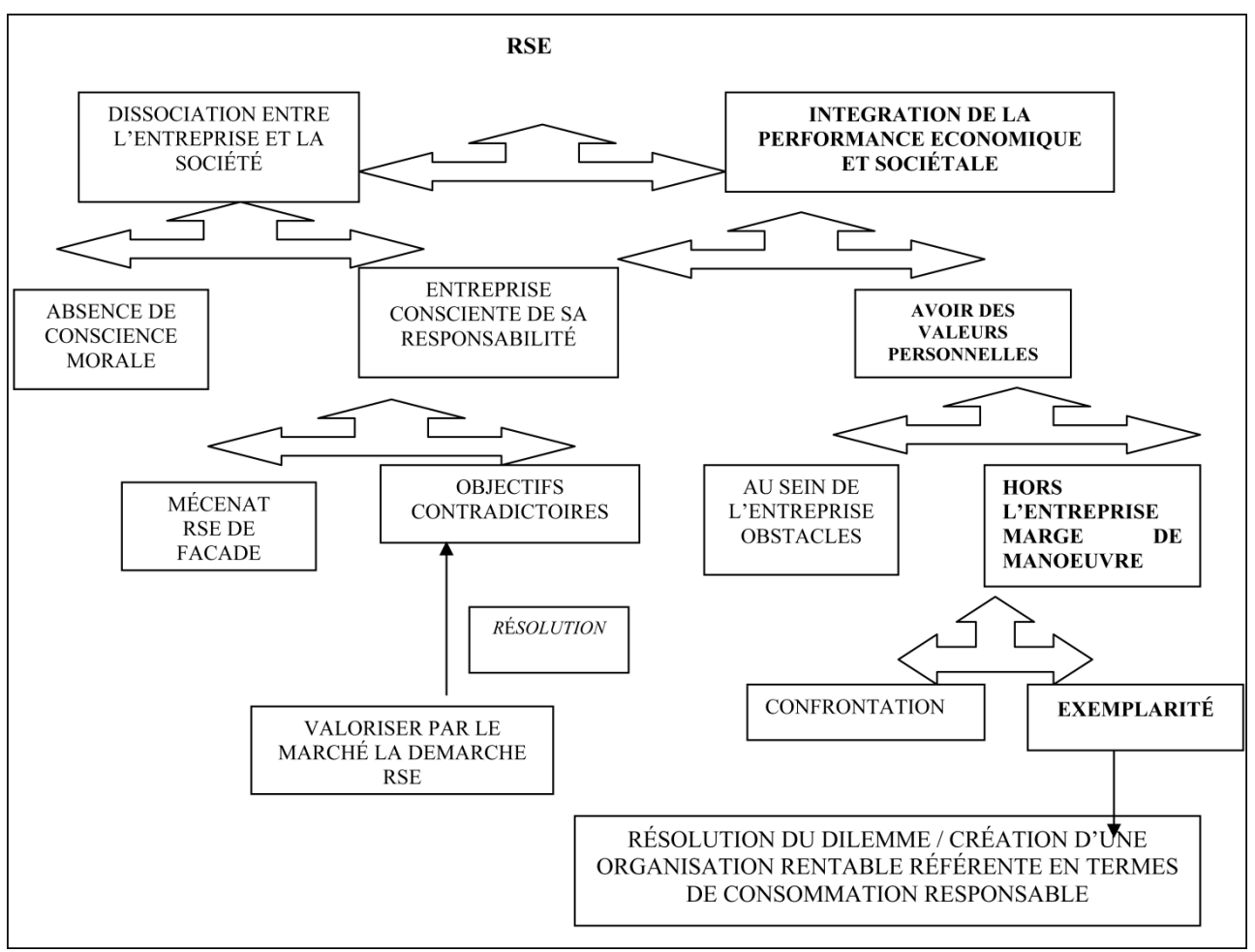

le bien public (l'environnement, les hommes). Dans cette optique, la démarche est inscrite dans le long terme et vise la cohésion sociale intra-entreprise et la cohésion entre l'entreprise et la société. La solution est alors pour EG de ne plus travailler dans les agences qui font de la publicité, mais de créer une structure, artisan de la communication.

La mise en place d'une démarche en RSE naît dans les quatre cas d'une situation de dilemme à résoudre. Ce dilemme peut être de source externe (écart entre le comportement de l'entreprise et le contexte réglementaire ou social, ou anticipation de risques de coûts pour l'entreprise), ou de source interne (tension entre les valeurs de l'individu et les pratiques de l'entreprise ou remise en question personnelle de l'activité professionnelle). La recherche de solutions est alors différente selon le contexte. Lorsque l'acteur agit pour le compte de l'entreprise et à sa demande, il doit trouver des solutions liées aux contraintes et aux ressources internes de l'organisation. Si la démarche est volontaire de la part des dirigeants, alors les acteurs apprennent, dans un processus d'essai-erreur, à mettre en place la RSE au travers d'échanges avec les parties prenantes internes et externes. Mais la RSE ne peut devenir une réelle opportunité à long terme que si elle s'accompagne 
Figure 4 - Schème de l'entretien 4 : la RSE, l'équilibre entre les acteurs sociaux

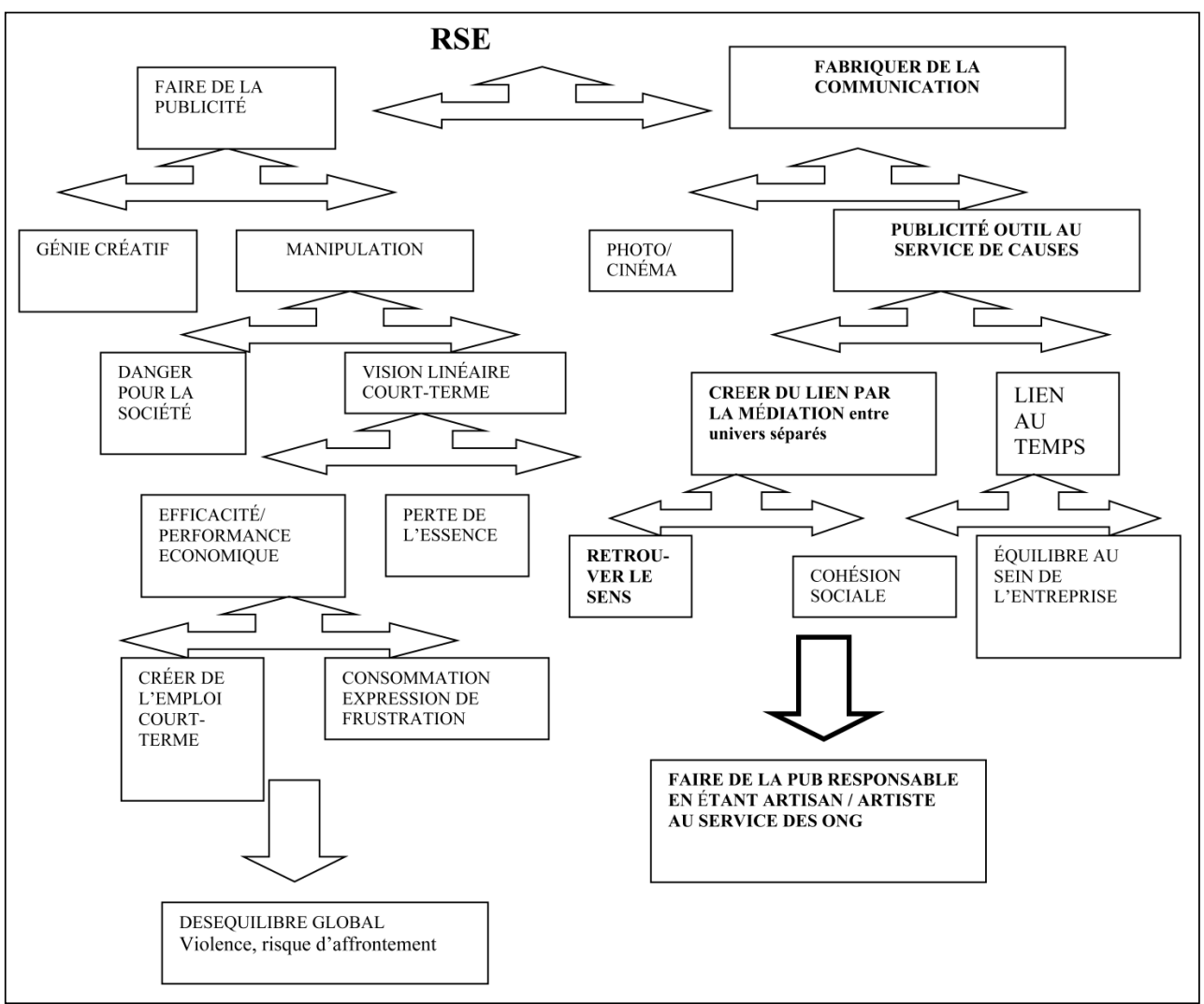

d'un changement de culture des acteurs. $A$ contrario, une vision à court terme risque d'engendrer une perte de contrôle et de performance économique (retrait de ressources, coûts plus lourds car non négociés, lois plus coercitives, perte de confiance des parties prenantes). Dans le cas d'une démarche individuelle, la solution ne peut pas être trouvée au sein de l'organisation et nécessite d'inventer un nouveau mode d'action pour les acteurs. L'issue dans les deux cas est trouvée au travers de l'établissement de liens avec des acteurs légitimes en termes de RSE, à savoir les associations et ONG qui savent faire. Dans tous les cas, les acteurs favorisent le partenariat à l'affrontement et définissent leur action dans le cadre du système actuel comme moyen de corriger des modes d'action déviants mais non pas coupables. Le principal levier pour encourager les comportements attendus est la valorisation économique de l'action en RSE.

Que signifient alors ces solutions apportées aux dilemmes vécus? D'abord, les acteurs semblent chercher à être moins aveugles à l'égard des conséquences de leurs décisions. Ainsi, dans les différentes situations étudiées, les acteurs discutent, testent, remettent en cause ou trouvent ailleurs des 
solutions quand par exemple l'organisation n'est pas prête à accueillir ce qui est considéré comme relevant de la RSE. Ils considèrent dans cet esprit l'exploitation de l'expérience comme une stratégie fructueuse. Cette démarche pragmatiste s'écarte de celle reposant uniquement sur l'intérêt égoïste éclairé puisqu'elle permet aux acteurs de considérer les problèmes sousjacents de l'organisation et de la société dans leur ensemble. Dans cette perspective, les organisations sont vues comme des moyens devant être utilisés pour des objectifs humains et non comme des propriétés devant être défendues.

La focalisation dans les cas étudiés sur la capacité humaine à comprendre et, dans certaines situations, à résoudre les problèmes et les tensions, ne doit pas être cependant confondue avec la notion de rationalité ou d'utilitarisme. Les expériences organisationnelles sont riches d'émotions tant négatives que positives, de comportements dits irrationnels, etc. de telle sorte qu'il n'est guère envisageable de rapprocher l'intelligence pragmatiste de la rationalité putative. Les émotions peuvent en effet améliorer la décision (Frank, 1988), et dans cette optique, elles peuvent être considérées comme un paramètre relativement important dans le contexte des expériences dans lesquelles les praticiens essayent de résoudre les problèmes rencontrés. L'utilitarisme (voir à ce sujet la critique de l'utilitarisme par Rawls, 1971) n'est pas non plus de mise dans la mesure où la valeur pragmatiste ne constitue pas « un pseudonyme de l'utilitarisme » (Wicks et Freeman, 1998). Les principes utilitaristes, quand ils sont appliqués aux organisations et à l'expérience sociétale, peuvent faire partie de l'approche pragmatiste à cause de leur centrage sur les conséquences. Vu dans cette optique, « l'utilitarisme... nécessite une évaluation rigoureuse, objective et impartiale des conséquences... Un outil puissant de raisonnement moral, c'est une technique qui vaut la peine d'être maîtrisée » (DeGeorge, 1990).

Cependant, les comportements dont les conséquences font l'objet d'évaluation et de jugement (Einhorn et Hogarth, 1978), sont associés quelque peu à des normes, générées dans un contexte organisationnel continu où les décisions sont liées aux problèmes quotidiens, ordinaires, issus des interactions organisations-environnement (ou entreprises-société). Les expériences passées, intégrant tant les succès que les échecs, forment un processus historique ou un continuum de pensée constitué des problèmes rencontrés. Ce processus produit des conventions et des traditions, accumulées historiquement, qui agissent comme des guides et des principes généraux pour l'exécution d'actions et la conduite des individus et des organisations vers les objectifs. Dès lors, les normes sont déterminées par l'expérience antérieure, mais atténuent en même temps la dépendance de sentier en intégrant l'information nouvellement générée. Ce processus, fondé sur l'expérience et résolvant des problèmes, est la source des valeurs opérationnelles et partant, le guide comportemental des praticiens.

Par ailleurs, comme la théorie des parties prenantes (Freeman, 1984; Goodpaster, 1991) nous apprend que le processus de formation des valeurs va au-delà des frontières de la firme, les problèmes que les organisations rencontrent, tout autant que les valeurs sous-jacentes aux décisions, sont le résultat des interactions firme-environne- 
ment, eu égard à un ensemble d'intérêts en tension (attentes, cultures, communautés, marchés, etc.). Partant, plus les intérêts de ces parties prenantes sont antinomiques, diversifiés et complexes, plus les valeurs résultantes le sont. La prise en compte de ces intérêts élargit la base expérientielle des acteurs et la quête pragmatique de résultats satisfaisants devient ardue parce qu'elle concerne toute une variété de valeurs (sociales, politiques, religieuses, ethniques, etc.), devant être incorporées dans les règles et les normes.

Ces normes et ces valeurs (parcours personnel, incidents, événements, éducation, etc.) sont, dans les quatre cas étudiés, socialement contextuelles, issues de l'expérience opérationnelle et utilisées comme guides décisionnels dans le but de résoudre des problèmes et de saisir des opportunités. Nous sommes loin ainsi d'individus ou d'organisations atomisés, faisant appel seulement à une conscience morale intrinsèque, mystique ou à une conception morale mythique et réifiée.

Cependant, comme dans la philosophie pragmatiste, les intérêts et les désirs se forment seulement en référence à des problèmes ou des situations problématiques, il est important d'expliquer ces derniers de peur que la théorie ne tombe dans le piège du dilemme utilitariste. Des dispositifs généraux sont nécessairement définis, mais la contingence et la construction de la situation doivent être identifiées - la contingence parce que les problèmes sont souvent les résultats non prévus des actions entreprises dans un monde incertain, et la construction parce que les institutions et les conventions aident à définir ce qu'est un « problème ». Les théories sociologique et évolutionniste nous apprennent que les acteurs, dans un environnement incertain, s'appuient sur des principes généraux et autres dispositifs sociaux pour structurer leur situation. Ce qui nécessite ici des conventions, entendues comme système d'anticipations mutuelles par rapport aux compétences et aux comportements des autres (Storper et Salais, 1997). Les conventions diffèrent des normes, dans la mesure où elles ne sont ni obligatoires ni explicitement contraintes. Ce sont des manières établies de faire les choses qui permettent aux individus de coordonner les anticipations. Pour les chercheurs académiques, les conventions permettent d'obtenir une certaine prise sur le problème considéré sans le définir totalement a priori. Quand les pratiques ne sont pas couronnées de succès (au regard des termes et des attentes des acteurs) dans un monde incertain, les acteurs envisagent de nouvelles voies pour construire la coordination.

Sur le plan des apports théoriques, cette recherche montre que la prise en compte de la RSE ne peut être réalisée sans référence à l'expérience réelle des parties impliquées il faut ainsi laisser de la place à la nouveauté. Dès lors, la situation (ou le cas) acquiert un pouvoir explicatif fort, mais ceci constitue une opportunité, non une menace. Un schéma pragmatique d'action encourage la théorisation à partir des problèmes, apte à traiter des contingences historiques et de l'incertitude, même si elle centre l'attention sur des ruptures probables des conventions. Dewey (1922, p. 236) estimait que « l'acceptation de fins fixées est un aspect de la dévotion de l'homme à l'idéal de la certitude ". Si celle-ci est ce qu'on exige des approches sociales, ce peut être troublant. Cependant, si nous acceptons que notre incapacité de tout savoir ne signifie pas que 
nous ne savons rien, nous permettons à la théorie « d'avancer comme mouvement à partir de la connaissance actuelle, plutôt qu'à travers une vérité absolue » (Sommers, 1998, p. 724), la science sociale peut être une entreprise de résolution des problèmes. Elle peut souligner, par exemple, que la coopération entre les acteurs est mieux saisie par la création d'institutions et de pratiques pour expérimenter conjointement de nouvelles solutions au lieu d'affirmer par exemple trivialement qu'il est impossible de rendre les firmes socialement responsables dans la mesure où leur objectif est avant tout financier, ou que la prise en compte des enjeux sociaux rend illégitimes les organisations sur le chapitre de la performance économique.

Sur le plan pratique, cette recherche fondée sur la quête de solutions a trois conséquences. La première a trait à l'émergence d'une double vision, sensibilisant les individus à des caractéristiques alternatives à une situation, multipliant les voies pour voir les faits et planifier les actions, même quand un engagement existe déjà pour certaines actions. Des objectifs, toujours en vigueur, aident les individus à reconnaître qu'ils ont des voies alternatives pour construire une situation.

La deuxième conséquence est que les objectifs RSE restent d'actualité même quand l'action engagée favorise un but au détriment d'un autre. Certaines décisions peuvent ne pas violer les critères RSE, ou du moins peuvent constituer les meilleure options disponibles à un instant $t$. Mais laisser cohabiter un certain nombre d'objectifs indique que la RSE doit être prise en compte même quand les considérations économiques prévalent, ce qui constitue un dépassement des travaux évoquées dans la première partie. Cette réflexion peut inciter les dirigeants à préserver, et peut être améliorer, les intérêts des parties prenantes non saillantes pour le moment. Quand la RSE est seulement une affaire de respect de droits parfaits, et d'évitement des choses non éthiques, elle n'est pas prise en considération tant que rien de mauvais ne s'est produit et qu'aucune ligne n'est transgressée. Mais lorsqu'un ensemble de buts doit être estimé, les dirigeants peuvent trouver le moyen de manipuler « le périphérique » à partir des buts subordonnés à un choix particulier.

La troisième conséquence est qu'avec l'existence de dilemmes ou d'objectifs en tension, les acteurs peuvent être poussés à inventer des voies créatives pour opérer au travers des problèmes enchevêtrés. Ce qui invite à de nouvelles façons d'envisager les situations et de nouvelles possibilités, générant ainsi des approches non conventionnelles pour aborder les conflits ; conflits qui existent même si la pensée stricto-économique tend à ignorer leur présence. Des recherches expérimentales (Higgins et Chaires, 1980) ont laissé entendre que les individus sont plus performants dans la résolution des tâches difficiles - quand ils sont conduits à voir les matériels à leur disposition non comme une boîte d'outils mais comme une boîte et des outils (Margolis, 1998). Les solutions constructives peuvent émerger quand les individus sont incités à identifier l'ensemble des options à leur disposition. Dès lors, organisations et « porteurs de RSE » peuvent trouver des solutions ensemble, les outils ne devant pas bloquer l'utilisation de la boîte. Similairement, la RSE ne doit pas être un obstacle à la mise en place de solutions pratiques, mais doit servir comme moyen de les utiliser ou de les compléter. 


\section{CONCLUSION}

Cet article a examiné les apports de la posture pragmatiste à l'étude de la RSE en s'appuyant sur l'utilisation des récits de vie comme outil méthodologique pour la prise en compte des phénomènes qualitatifs, processuels et longitudinaux. La recherche a mis en évidence la pertinence de cette posture à la compréhension de la façon dont les acteurs construisent les réponses à apporter aux problématiques de la RSE. Celle-ci est le résultat de l'interaction des acteurs, qui s'appuient sur leurs expériences passées et présentes pour développer les solutions requises. Les situations traitées émergent alors avec un pouvoir explicatif significatif, elles sont en même temps susceptibles de servir de guides normatifs aux problèmes à venir pour des situations identiques. L'analyse proposée est ainsi novatrice dans le champ de la RSE dans la mesure où, se situant dans une perspective distincte des approches théoriques majeures en RSE, elle fait des individus et des organisations des acteurs non atomisés, sociaux, en interaction continue, réfléchissant d'une manière créative et concrète aux situations auxquelles ils sont confrontés. Cette analyse révèle en même temps des potentialités intéressantes pour comprendre d'une manière concrète les problèmes vécus par les acteurs dans d'autres domaines des sciences de gestion.

En prenant comme cadre de référence le pragmatisme, l'intérêt de ce travail aura été de mettre en évidence le rôle moteur de la RSE pour stimuler les acteurs à s'engager dans une meilleure compréhension de l'interaction entre la société et l'organisation et pour rechercher des solutions créatives tant en termes organisationnels, qu'en termes de positionnement de marché ou d'innovations produits. Dès lors, la tension entre faire des profits et servir la société n'est plus de mise et la réflexion ne vise ni l'invalidation, ni la réconciliation des objectifs antinomiques. Cette recherche mériterait cependant d'être enrichie par un recueil d'informations complémentaires auprès d'autres acteurs ayant participé au processus de résolution des problématiques en RSE, notamment les individus réfractaires dans le but d'évaluer le type de chemin utilisé dans ce cas pour éviter ou retourner le processus de mise en œuvre de la RSE. On pressent en effet dans le discours des interviewés l'importance d'un ensemble d'acteurs ayant joué un rôle de freins dans le développement des pratiques en RSE. Ce regard plutôt « négatif » à l'égard de la RSE accroîtrait la compréhension des modes d'action et des motivations pouvant contrer le développement de la RSE au sein des organisations. Les résultats obtenus seraient également améliorés en ayant recours à un échantillon d'individus concernés par d'autres problématiques ou à d'autres types d'acteurs. En outre, la méthode des récits de vie, aussi riche soit-elle, n'évite pas le biais de désirabilité sociale et de rationalisation $a$ posteriori de l'interviewé. Une analyse plus aboutie des processus psychologiques déployés par l'interviewé permettrait d'améliorer la compréhension du discours. Cette analyse relève cependant, comme le soulignent Demazière et Dubar (1997), du domaine psychanalytique.

La comparaison des quatre schèmes permet l'identification des entités noyaux, ce qui pourrait constituer ensuite les concepts types traduisant la démarche des acteurs de construction de la RSE. 
Figure 5 - Concepts types de la démarche de construction de la RSE

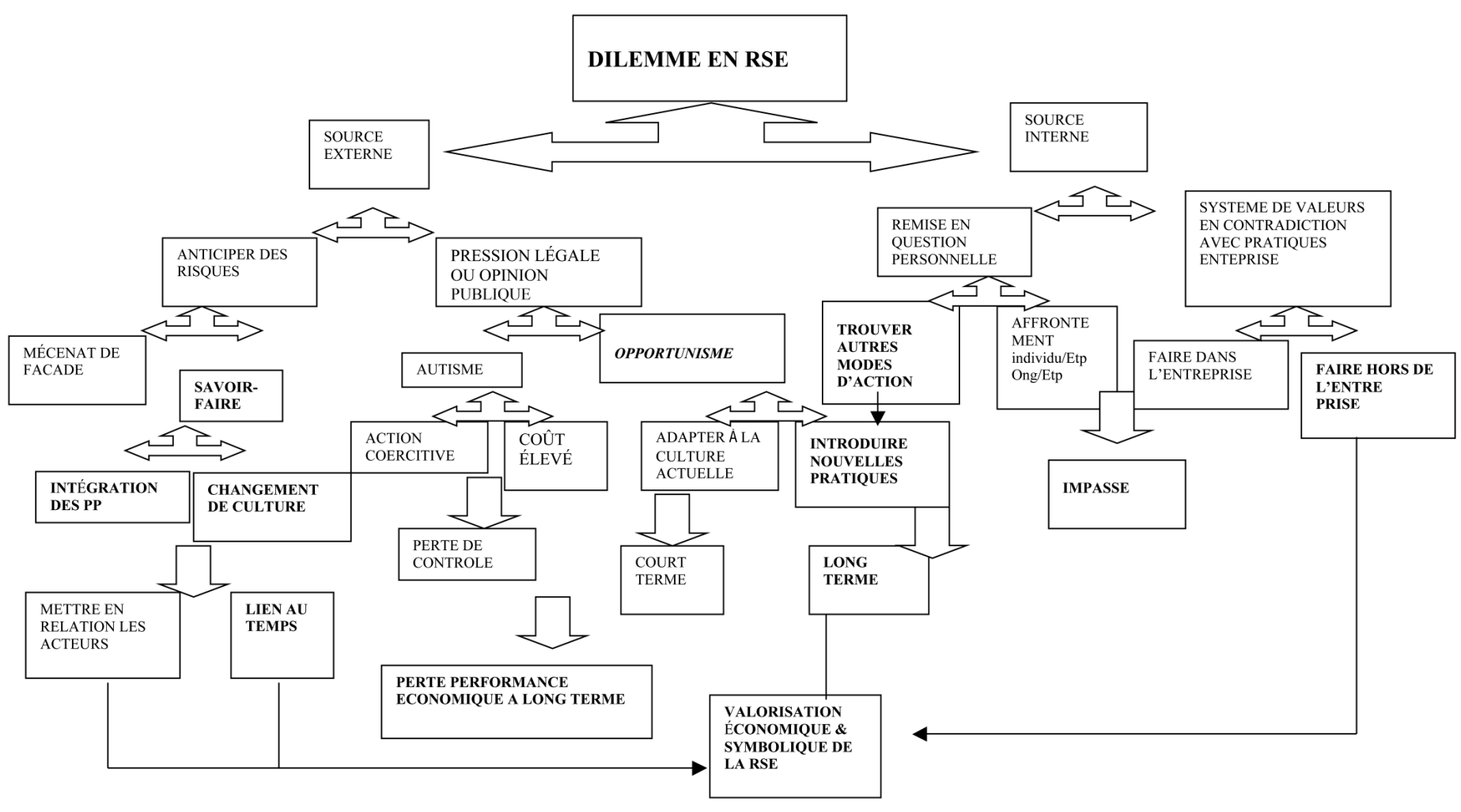




\section{BibLIOGRAPHIE}

Alexander J. C., "The New Theoretical Movement", Handbook of Sociology, N. J. Smelser (Ed.), Newbury Park, CA, Sage, 1988.

Bentham J., Déontologie, ou science de la morale, Tome 2, traduction française, Paris, Charpentier, 1834.

Bertaux D., Les récits de vie, Paris, Nathan, 1997.

Buchholz R. A., Rosenthal S. B., "Business and Society: What's in a Name”, The International Journal of Organizational Analysis, vol. 5, $\mathrm{n}^{\circ}$ 2, 1997, p. 180-201.

Buchholz R. A., Rosenthal S. B., "A Philosophical framework for Case Studies”, Journal for Business Ethics, vol. 29, n 1/2, 2001, p. 25-31.

Carroll A. B., "A Three-Dimensional Model of Corporate Performance", Academy of Management Review, vol. 4, n 4, 1979, p. 497-505.

Carroll A.B., "Managing Ethically with Global Stakeholders: A Present and Future Challenge, Aacdemy of Management Executive, vol. 18, n 2, 2004, p. 114-120.

Christian D., Boudès T., « Vers une stratégie narrative, il était une fois la stratégie, Revue française de Gestion, ${ }^{\circ}$ 120, septembre-octobre 1998, p. 110-119.

Daft R. L., Weick K., "Toward a Model of Organizations as Interpretation Systems", Academy of Management Review, vol. 9, $\mathrm{n}^{\circ} 2$ 2, 1994, p. 284-295.

De George R., Business Ethics, New York, MacMillan, 1990.

Demazière D., Dubar C., Analyser les entretiens biographique: l'exemple de récits d'insertion, Paris, Nathan, coll. " Essais et Recherches », 1997.

Dewey J., Reconstruction in Philosophy, New York, Henry Holt, 1920.

Dewey J., Human Nature and Conduct, New York, The Modern Library, 1922/1957.

Dewey J., Experience and Nature, New York, Dover, 1958.

Donaldson T., "Making Stakeholder Theory Whole", Academy of Management Review, vol. 24, n², 1999, p. 237-241.

Einhorn H. J., Hogarth R. M., "Confidence in Judgement: Persistence of Illusion of Validity”, Psychological Review, 85, 1978, p. 395-416.

Frank R. H., Passions Within Reason: The Strategic Role of the Emotions, New York, W W. Norton, 1988.

Freeman R. E., Strategic Management: A Stakeholder Approach, Boston, Pitnam, 1984.

Friedman M., Capitalism and Freedom, Chicago, University of Chicago Press, 1962.

Gond J.-P., Mercier S., Les théories parties prenantes : une synthèse critique de la littérature, université de Toulouse, notes du LIRHE, Note $n^{\circ} 411,2005$.

Giroux N., «L'analyse narrative de la stratégie », Actes du IX Congrès de l'AIMS, Montpellier, 2000.

Goodpaster K.E., "Business Ethics and Stakeholder Analysis", Business Ethics Quarterly, 1, 1991, p. 53-73.

Griffin J.J., Mahon J.F., "The Corporate Social Performance and Corporate Financial Performance Debate", Business and Society, vol. 36, n 1, 1997, p. 5-31. 
Higgins E. T., Chaires W.M., "Accessibility of Interrelational Constructs: Implications for Stimulus Encoding and Creativity", Journal of Experimenal Social Psychology, 16, 1980, p. 348-361.

James W., Pragmatism and the Meaning of Truth, Cambridge, MA, Harvard University Press, 1975/1907.

Jensen M. C., "Value Maximization, Stakeholder Theory and the Coprporate Objective Function", Business Ethics Quarterly, vol. 12, n² 2, 2002, p. 235-256.

Joas H., Pragmatism and Social Theory, Chicago, University of Chicago Press, 1993.

Joas H., The Creativity of Action, Chicago, University of Chicago Press, 1996.

Legrand J.-L., « Glossaire commenté», Les Histoires de vie, tome II, Pineau G., Jobert G. (Ed.), Paris, L'Harmattan, 1989.

Margolis J., "Psychological Pragmatism and the imperative of Aims: A New Approach for Business Ethics", Business Ethics Quarterly, vol. 8, n³ 3, 1998, p. 409-430.

Rawls J., Theory of Justice, traduction française: Théorie de la justice, Paris, Le Seuil, coll. « Points», 1971.

Solomon R. C., Ethics and Excellence: Cooperation and Integrity in Business, New York, Oxford University Press, 1992.

Stern R. N., Barley S. R., “Organizations and Social Systems: Organization Theory's Neglected Mandate", Administrative Science Quarterly, 41, 1996, p. 146-162.

Storper M., Salais R., Worlds of Production: The Action Frameworks of the Economy, Cambridge, Harvard University Press, 1997.

Wicks A.C., Gilbert D. R., Freeman R.E., "A Feminist Reinterpretation of the Stakeholder Concept", Business Ethics Quarterly, vol. 4, n 4, 1994, p. 475-497.

Wicks A.C., Freeman R.H., "Organization Studies and The New Pragmatism: Positivism, Anti-Positivism, and the Search for Ethics", Organization Science, vol. 9, n 2, 1998, p. 123-140. 